\title{
Off-line washing effectiveness on a multistage axial compressor
}

\author{
Nicola Aldi, Nicola Casari, Ettore Fadiga, Riccardo Friso, Stefano Oliani, Michele Pinelli, \\ Pier Ruggero Spina, Alessio Suman*, Alessandro Vulpio and Nicola Zanini \\ University of Ferrara - Department of Engineering, via Saragat, 1, 44122 Ferrara, Italy
}

\begin{abstract}
The interaction between gas turbines and airborne particles determines detrimental effects on the performance, efficiency, and reliability of the power unit. When it is possible, the interaction is reduced by the use of inlet separators and filtration systems. In an aero engine, these barriers are difficult to implement, and only bigger particles (usually greater than $10 \mu \mathrm{m}$ ) are separated from the airflow. Small units, especially those equips helicopters, are usually affected by fouling issues, especially when the aircraft is employed in harsh environments such as firefighting and rescue activities. To recover this contamination, the unit is washed after the mission by ground operations to restore the unit performance by removing the deposits. This operation occurs during a sub-idle unit operation, and the washing process has to be effective when the engine operates in this off-design condition. In this paper, the evaluation of the washing performance during a sub-idle unit operation is carried out. The compressor unit is a multistage axial compressor that equips the Allison 250-C18 engine. The washing operation was performed by water, and a sensitivity analysis is carried out to discover the capability of water droplets to remove the contaminants. The experimental analysis involves the contamination of the unit by micro-sized soot particles and a washing operation by micro-sized water droplets. These experimental results are compared to numerical simulations to discover the effects of the washing operation on a small power unit during sub-idle operating conditions. The off-design regime imposes a specific evaluation of the proper setup of the washing strategy: flow separations involve wider regions in the compressor unit, and the removal capability is strongly related to the droplet path through the stages. The results show how in the off-design washing operation, the droplet diameter has greater importance than the water flow rate for reducing the deposits over the compressor stages.
\end{abstract}

\section{Introduction}

Particle adhesion, commonly known as fouling, characterizes several issues in the numbers of machines and energy system devices as the heat exchanger, solar panels, and gas turbines $[1-4]$. The interaction between micro/nano-sized solid particles and the relevant surface of the machine determines the modification of the surface characteristics $[5,6]$ or,

\footnotetext{
* Corresponding author: alessio.suman@unife.it
} 
in the worst cases, the modification of the shape and the physical characteristics (such as the conductivity) determining detrimental effects in terms of reliability and safety.

Looking at the gas turbine field, the operation regime, and the conditions of the environment determine the development of the fouling effects and the severity of the performance losses. The concentration of natural and/or artificial micro-sized particles, together with the air humidity, are those of the most critical variables on the fouling rate [7]. Literature data have revealed that the fouling issue affects the unit performance despite the presence of filtration systems $[8,9]$ and, only with a periodic (frequent) online washing operation $[5,10,11]$, the reliability of the engine remains acceptable.

A particular harsh condition experienced by a gas turbine is related to the helicopter operation. These engines usually operate in dusty conditions due to the downwash of the rotor that spreads microparticles from the ground into the environment (up to a few meters), as reported in [12]. In addition to this, a helicopter operation is mainly localized at low altitude $(<2000 \mathrm{~m})$, where the presence of nano/microparticles coming from the combustion process are present considerably [13]. These unlucky conditions imply proper management of the helicopter engine, especially in terms of daily maintenance and ground operation after each mission. Deposits located at the compressor section have to be removed by a washing operation usually carried out by manual operation, using a manual jet during a subidle operation regime of the engine immediately after the mission. This operation could be carried out using water or cleaner depending on the location and resource availability [14].

Gas turbine washing operations have been extensively studied to evaluate the effectiveness according to the operation regime, water flow rate, and droplet diameter [10, $11,15]$. These data have demonstrated how, for online washing operations, by increasing mass flow rate and droplet diameter, the effectiveness of the washing operation increases. The upper limits of these parameters are the water or cleaner consumption (and thus costs) and the blade damage in terms of erosion issues due to the droplet impingement [9]. These findings are obtained by dedicated experiments [15] and gained by on-field experiences and detection related to the land-based units [5]. Regarding the aero-engine, only a few contributions are present in literature [11], even considering the particular conditions under which the helicopter engine is cleaned.

In light of these considerations, in this paper, a detailed analysis of the washing operation was carried out on a helicopter unit. The multistage axial compressor was fouled by micro-sized soot particles, and then it has been washed during a sub-idle operating condition. The experimental analysis, based on the modification of water droplets (three different diameters), has been coupled to the numerical analysis that allows the interpretation of the experimental results. The washing effectiveness has been evaluated employing image analysis, considering the cleanness of the blade and vane surface by comparing the clean, fouled, and washing images.

\section{Experimental apparatus}

The test bench mounts the compressor of the engine Allison $250 \mathrm{C} 18$. For this engine, the multistage axial unit is coupled with a centrifugal stage for an overall pressure ratio of 6.2 and a mass flow rate of $1.36 \mathrm{~kg} / \mathrm{s}$ at the design speed of 51,600 rpm [16]. According to Fig. 1 , the test bench is equipped with an external air treatment section, which allows increasing the relative humidity at the compressor inlet (by a water steam injector), and a particle injection system, able to contaminate with a precise and controlled amount of soot particles the airflow swallowed by the unit. The contamination section is positioned 2-m far from the compressor inlet (ensuring the kinematic equilibrium between powder and airflow as well as the uniform particle distribution at the compressor inlet section), and it is based on the particle feeder TOPAS SAG40U that prepares and injects the solid contaminants by a two Venturi eductors which guarantee the proper soot particle injection process (the powder is 
prepared, deagglomerated, and dosed by the first Venturi nozzle, while the second one, mounted inside the compressor inlet pipeline, injects the powder contaminating the airflow). The dosing system and the setup of the contaminant injection are described in [17].

In addition to the air inlet treatment devices, the compressor inlet section mounts a nozzle ring $0.3-\mathrm{m}$ far from the compressor inlet. This nozzle ring is composed of four injection points that generate full cones radially to the main airflow. A regulation valve managed the pressure of the water, and a volumetric pump supplied the water. The nozzles can be easily dismounted, cleaned, and changed to test different droplet diameter distributions and water flow rates. A detailed description of the nozzle setup is reported in [14].

To detect the removal capability of the washing procedure, a set of images of the compressor flow path has been taken during the experimental investigation. A proper light and camera setup was adopted to ensure the repeatability of the detection process that allows the comparison between the different removal capabilities of the different washing strategies adopted in the present work. The image detection was carried out by disassembling the compressor unit. Thanks to this, the half compressor case (stator) was positioned on a realized-on-purpose holder while the rotor was kept in the original position. The deposit patterns are taken directly from the lab-testing facility employing dedicated cameras (Canon EOS M6 mirrorless digital camera with Tokina $100 \mathrm{~mm}$ lens with a resolution of $3552 \times 2664$ pixels) positioned to detect the entire flow path of the axial compressor divided according to the rotor and stator components. The white light setup is adopted for both parts of the compressor flow path with the intensity of $4000 \mathrm{~K}$. Starting from these detections, the determination of the deposit pattern has been carried out through image processing based on the open-source image processing package named IMAGE $\mathbf{J}$ [18]. The image post-process has been carried out by the following procedure. The clean picture has been subtracted to the fouled and washed one for all the conditions tested. For

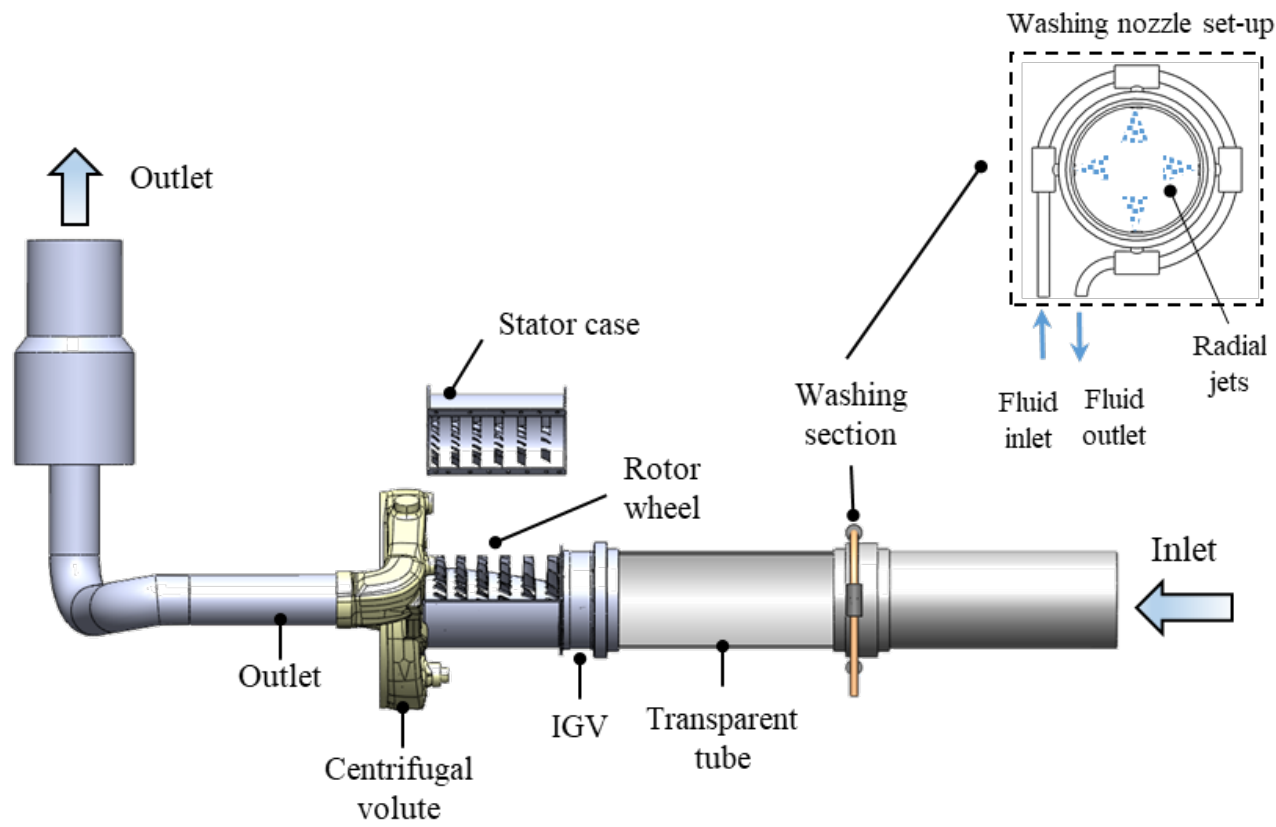

Fig. 1. Experimental apparatus for the experimental assessment of compressor fouling and washing 
each tested condition, two pictures have been generated for both rotor and stator: one that highlights the patterns after the particle ingestion and the other that highlights the residual deposit after the washing process. By comparing the pictures coming from this subtraction, the region affected by deposits and the regions which are cleaned by the washing procedure can be easily compared. More details about experimental strategy are reported in [19-21].

\subsection{Experimental procedure}

The characterization of the removal capability is carried out considering a fouling process due to soot particles. Soot particles, supplied by Powder Technology Inc. are constituted by carbon. The mean particle diameter is equal to $1.9 \mu \mathrm{m}$, while the detailed characterization of the powder is reported in [14]. The shape of soot particles allows the creation of a stable and coherent layer [22] useful to detect the removal capability of the washing strategy. The number of injected particles per unit of time was kept constant (336 million particles per second). Before being injected, the powder has been baked for $12 \mathrm{~h}$ at $70{ }^{\circ} \mathrm{C}$ to eliminate the moisture. Thanks to the installation of the steam humidifier, the deposition tests have been carried out increasing the relative humidity values up to $80 \% \mathrm{RH}$. This value is kept constant during the exposure time, avoiding the condensation phenomenon at the compressor inlet section. The compressor unit was operated at 20,000 rpm (sub-idle condition) during both contamination and washing procedures. Since detachment phenomena could take place, an exposure time of 30 min was chosen [23, 24].

Regarding the washing procedure, the tests have been carried out by fixing the total consumption of cleaning fluid $(0.305 \mathrm{l} /$ test $)$, allowing a direct comparison among the results. The test conditions are reported in Tab. 1: three different nozzle setups (three droplet diameters) have been adopted, and, to ensure the same amount of consumed water, the duration of the washing procedure was adjusted. The adjustment allows keeping constant also the water-to-air ratio $\left(w_{\text {tar }}\right)$ fixed at 1 according to the literature investigations [15]. At the end of the fluid injection, the compressor runs for three minutes to eject all the liquid from the compressor.

Table 1. Experimental matrix related to the washing parameters

\begin{tabular}{|c|c|c|c|}
\hline $\begin{array}{c}\text { Mean droplet diameter } \\
{[\boldsymbol{\mu \mathbf { m } ]}}\end{array}$ & $\begin{array}{c}\text { Water flow rate } \\
{[\mathbf{1} / \mathbf{m i n}]}\end{array}$ & $\begin{array}{c}\boldsymbol{w}_{\text {tar }} \\
{[-]}\end{array}$ & $\begin{array}{c}\text { Washing duration } \\
{[\mathbf{s}]}\end{array}$ \\
\hline 100 & 0.195 & 1.02 & 93 \\
\hline 50 & 0.198 & 1.03 & 92 \\
\hline 20 & 0.192 & 1.00 & 95 \\
\hline
\end{tabular}

The entire experimental procedure is a result of several steps, defined for ensuring the repeatability of the fouling and washing assessment:

- stator case disassembling, hand-cleaning, and the picture of the stator vanes and rotor blades in clean conditions are taken;

- stator case reassembling;

- compressor run for reaching the stable thermal condition (air temperature value at the compressor discharge section) and the proper relative humidity value at the compressor inlet (80\%RH);

- compressor fouling: $30 \mathrm{~min}$ of compressor operation at 20,000 rpm (corrected rotational speed) with constant particles ingestion (336 million particles per second);

- stator case disassembling and the picture of the stator vanes and rotor blades in fouled condition are taken;

- stator case reassembling; 
- compressor run for reaching the stable thermal condition (air temperature value at the compressor discharge section);

- compressor washing: according to the test matrix reported in Tab. 1, the compressor was washed for the prescribed time;

- compressor run for the drying process (2 min);

- stator case disassembling and the picture of the stator vanes and rotor blades in washed condition are taken.

\section{Compressor numerical model}

The present investigation is based on the numerical model and results reported in [25]. The numerical model comprises the six axial stages, modeled according to a single blade passage for each wheel. The details of the CFD model are extensively reported in [25], and it has been used in $[26,27]$ for extracting information related to the solid particle impact on the blade and vane surfaces. In the present paper, a similar approach has been adopted. A multiphase simulation was carried out using the Eulerian conservation equations for the continuous phase (air) and a Lagrangian frame to simulate a discrete second phase (water droplets). After the gas flow field simulation, the trajectories of individual water droplets were tracked by integrating a force balance equation on the particle. All the details related to the multiphase numerical approach can be found in [25 - 27]. A total of 500,000 representative droplets were injected at the compressor inlet section using a uniform diameter according to the data reported in Table 1. The water droplet tracking allows predicting the coordinates, velocity, and angle of the impacts on blade and vane surfaces. Since the investigated water droplet behavior refers to a micro-sized range, no break-up effects were considered. Therefore, the first impact of the water droplet was recorded, and the impact map on the relevant surface was compared to the experimental investigation of clean and fouled zones after the washing procedure. Therefore, it is possible to show the impacts of the uncontaminated droplet to detect the effects of removal and re-deposition.

\section{Results}

According to the experimental procedure and the numerical model early described, the analysis of the washing phenomena is reported, respectively. The multistage axial compressor was operated at the sub-idle condition (the idle condition of this unit is approximately $32,000 \mathrm{rpm}$ ) in the common regime during which the washing procedure was carried out (ground manual operation). The comparison between the experimental and the numerical results explains in detail the removal effects provided by water droplets.

\subsection{Experimental tests}

Figure 2 reported the greyscale images obtained by the post-process based on detecting the clean, fouled, and washed conditions for the stator vanes and rotor blades. The greyscale colored pattern represents the pixel-by-pixel difference between the picture taken before and after the deposition test/washing operation concerning the clean one, and in turn, represents the fouled region of the compressor flow path. The pattern called "fouled" is representative of the compressor contamination after $30 \mathrm{~min}$ of exposure time carried out with soot powder. The patterns called "washed" represent the difference between the clean detection and the detection carried out after the washing procedure, according to the three different parameters (in terms of droplet diameter, see Table 1 for more details). From the visual inspection of the unit, no erosion and abrasion issues were discovered over the deposition and washing tests. 
About the fouled condition, the contamination appears distributed over the entire compressor flow path, and even the rear stages are affected by particle deposition. The powders' micro-sized dimension determines the particle's capability to penetrate through the stages involving the entire blade surface. Therefore, no specific actions provided by the centrifugal force have been noticed. The detections show that the contamination is higher for the pressure side of the stator and rotor vanes and blades. The suction side is involved in the deposition process only for the region located at a higher radius, especially in the stator vanes detection. Moving to the washed patterns, several differences can be highlighted. In this case, the grey-colored pixels represent the contaminants that are not be removed by the washing droplet. Therefore, comparing the fouled patterns with the washed ones it is possible to detect two different mechanisms related to (i) the removal capability of the droplets (the same region becomes lighter from the fouled to the washed condition) and (ii) the re-deposition process due to the soot particles carried by the water droplets (the same region becomes darker from the fouled to the washed condition).

Looking at the post-processed figure, the deposit patterns show that the smallest droplets appear to be more effective for removing the soot deposits over the compressor stages, especially in the pressure side of the stator vanes. The first stages appear lighter in the case of the smallest droplet. In contrast, the rear stages have similar patterns, even a greater re-deposition can be noticed for higher droplet diameters for both pressure and suction side in the stator vanes detections. Looking at the rotor blade, bigger droplets appear to be effective for the first stages, especially looking at the pressure side and the blade leading edge. In the case of the rotor surface, the conditions of the rear stage appear similar over the investigated matrix. State the present test condition, where the compressor operational regime has been kept in off-design conditions, the mean droplet diameter, which leads to the best results in terms of surface cleaning, is the smallest one. This result is counterintuitive with respect to the open literature. For example, in [15], the washing conditions that led to the best engine recovery involved droplets with a coarse diameter, but the engine has been kept at a full load regime when the washing procedure was carried out. In the present application, the reduced rotational speed, which brings the compressor to work in off-design conditions, is responsible for significant modifications of the flow path, which are analyzed in the following section, thanks to the numerical simulation of the unit.

\subsection{Numerical analysis}

The multiphase numerical simulation allows the representation of the internal flow phenomena, especially in the present case, where the unit was operated in off-design conditions. The present numerical simulation does not account for the interaction between droplets and deposits (removal mechanisms are dependent on the physical and chemical interactions between the water droplet and deposited layer), but it helps to define the droplet path-line and find the impact region on the compressor blade and vane surfaces. Based on the numerical results, several considerations can be made concerning the cleaned regions and the re-deposition phenomena.

Figure 3 reports the impact location of the water droplets, highlighted according to their impact velocity. The first findings coming from the numerical simulation are related to the capability of smaller particles to flow through the compressor unit. Bigger particles appear only on the IGV and first rotor surfaces, while the smallest droplets involve all the stages.

This result is the direct consequence of the simulation setup. As reported in the previous section, the numerical analysis shows the first impact between each water droplet and the compressor surface to detect which regions are involved by the impact of uncontaminated droplets. Looking at Fig. 3, it is possible to highlight that a more significant amount of uncontaminated smaller droplets can reach the rear stages instead of bigger droplets, which are localized on the IGV and the first rotor. It is evident that the washed pattern shown in 

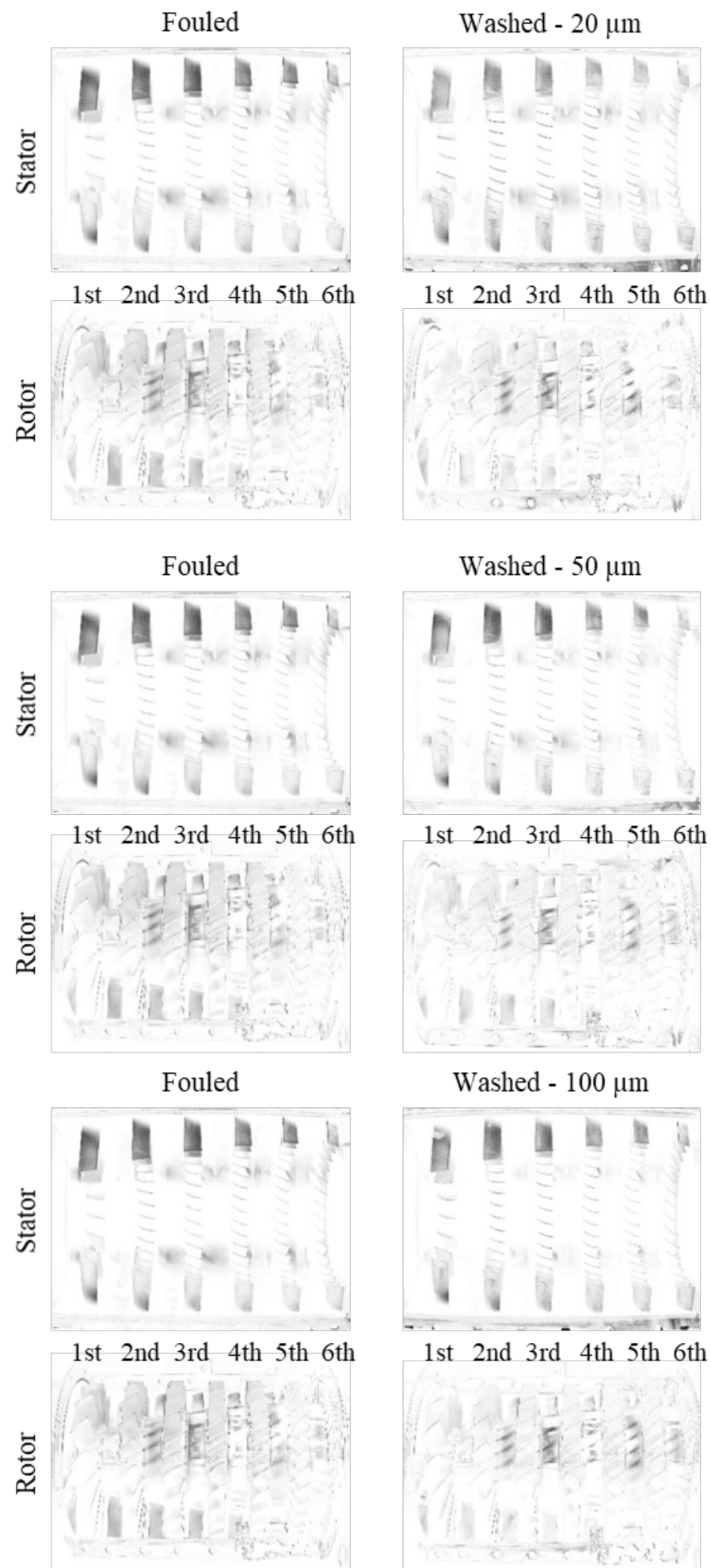

Fig. 2. Post-processed images of the stator vanes and rotor blades (six stages) deposits in the fouled condition (pre-washing) and after the washing procedure for the three droplets diameter sizes. 

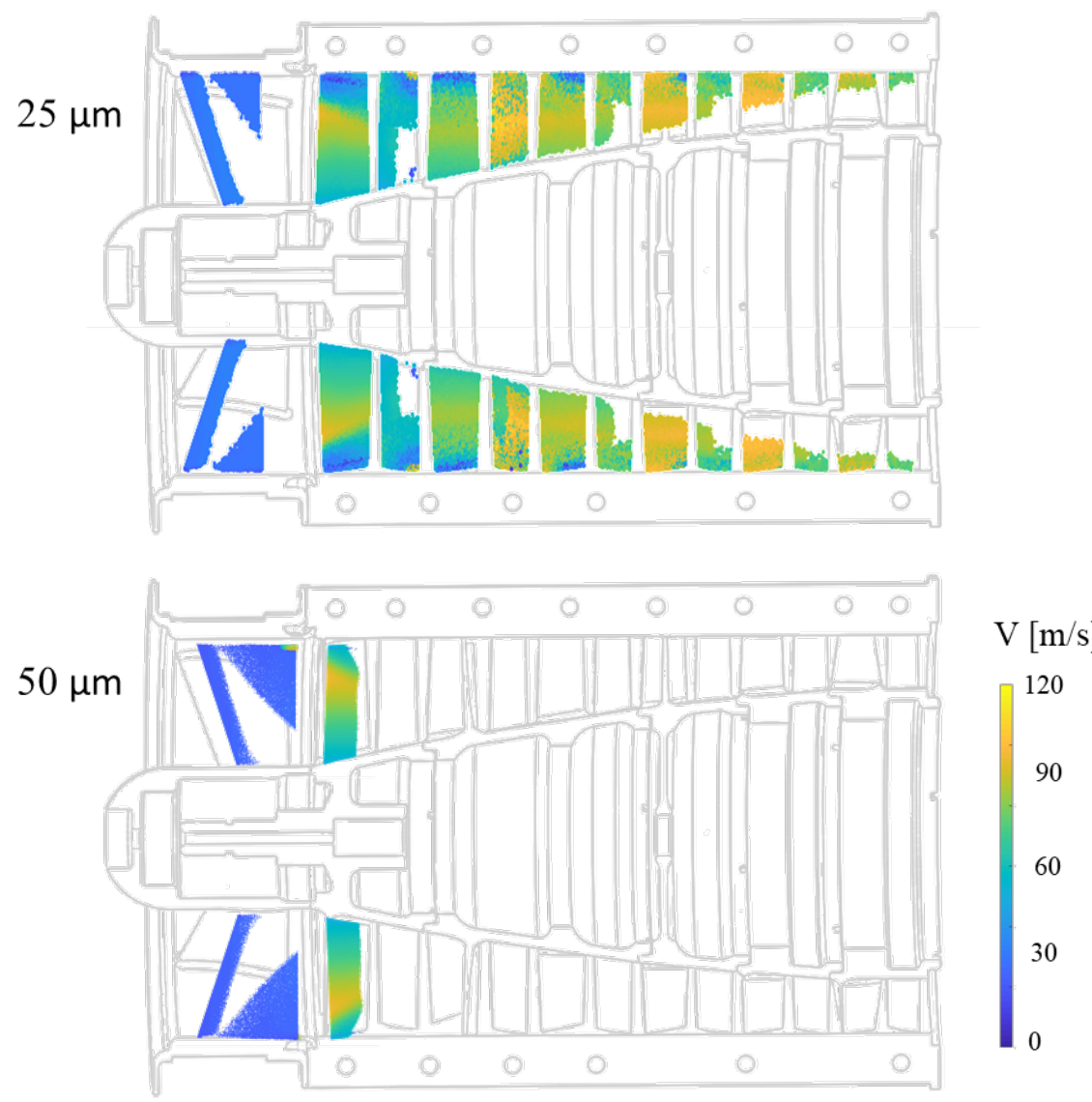

$\mathrm{V}[\mathrm{m} / \mathrm{s}]$

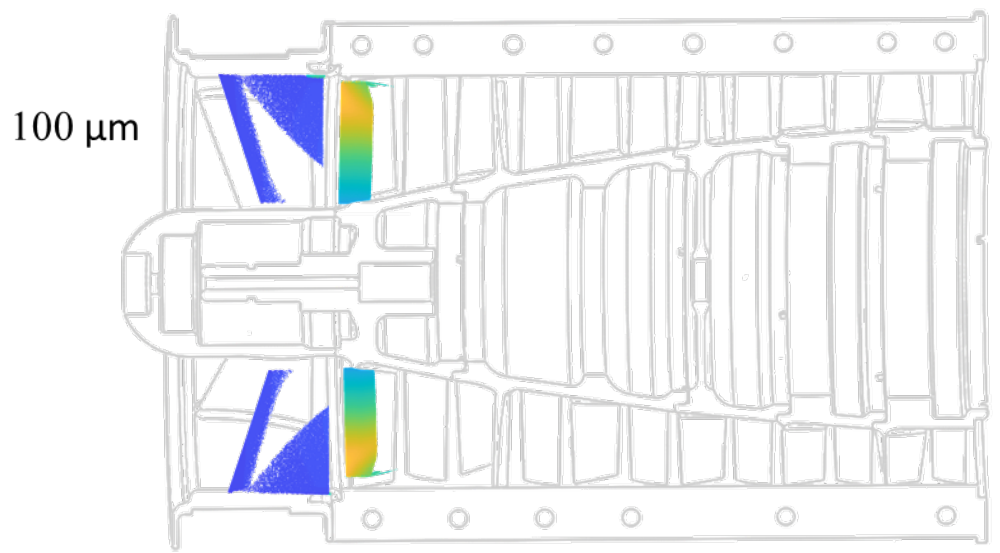

Fig. 3. Water droplets impact velocity over the compressor stages for the three investigated diameters

Fig. 2 is a result of the impact process carried out by the uncontaminated and contaminated droplets resulting from a previous impact over the compressor stages, but at the same time, this analysis puts the attention on the effects of a re-deposition process that could affect the rear stage. When the compressor operates in an off-design regime, the incident angle and the separation phenomena characterized the entire flow path predicting the washing operation not-straightforward. Therefore, by using bigger droplets for cleaning a 
compressor that operates in off-design conditions (a typical sub-idle condition realized during ground operations), the rear stages are affected by the impacts generated by a contaminated water droplet, coming from the break-up process carried out in the first stages which reduce the diameter, but at the same time, determines the contamination of the resulting droplets which are prone to determine re-deposition at the second impact. Even the major detrimental effects due to the fouling process are due to the front stages [11], the re-deposition process could be detrimental for both performance and corrosion issues that could be started after the washing procedure if the rear stage is not adequately washed or rinse.

Beyond the considerations related to the entire compressor flow path, differences in the water droplet diameter also evaluate the different impacting processes between the pressure side and suction side. Due to the off-design operating regime, wider separation zones are generated in correspondence with the airfoil surfaces (especially on the suction side surfaces), as reported in Fig. 4 according to blade-to-blade plots for five-blade spans. The flow separation zones are located mainly on the suction sides of the airfoils, near the compressor case, and to a minor extent on the pressure sides of the first stage airfoil. The spread of the separation zones seems to reduce towards the aft stages of the compressor. Small droplets, characterized by diffusive-inertial mechanisms of motion, can cross the separation zone and reach the airfoil surface, while the high inertia of coarse droplets denies this behavior. Moreover, for a fixed mass flow rate, the number of droplets that hit the surfaces is higher in the case of small droplet diameters, leading to high surface coverage for this washing condition.

State the present experimental and numerical analyses, some guidelines can be drawn concerning the off-line washing effectiveness on a multistage axial compressor. Despite the results reported in the open literature $[10,11]$, specific applications, as compressor units on aircraft or helicopters, are characterized by operating regimes far from the designed one and the expected effects studied for the in-design regime of operation differ dramatically. In particular, the incident angle determines a considerable amount of impact in the pressure side effects, determining a droplet break-up and, with the consequent modification of the droplet path-lines and the possible contamination of the water which arrives in the rear stage after several impacts. Therefore, for the off-design regime of operation, smaller droplets appear to be effective for removing the deposits over the entire compressor flow path [28], and at the same time, reducing and avoiding possible erosion phenomena, which are related to the dimension of the droplets [9].

\section{Conclusions}

The present investigation has reported an experimental and numerical investigation on the off-line washing effectiveness of a multistage axial compressor. The test matrix has been defined to detect the influence of the water droplet dimension on the removal capability of the entire washing process. The off-design conditions commonly adopted for washing the aircraft and helicopter engines increase the issues related to define the best procedure.

The washing process has been carried out with three droplet size distributions (with a mean diameter of $20 \mu \mathrm{m}, 50 \mu \mathrm{m}$, and $100 \mu \mathrm{m}$ ) to include the influence of the washing parameters on the assessment of the cleaning effectiveness. All the tests have been carried out employing the same water-to-air ratio, equal to $1 \%$, to compare the effects of droplet size in a precise way. The cleaning effectiveness has been estimated through image postprocessing methods applied to the stator vanes and rotor blades, comparing the detection of clean, fouled, and washed compressor surfaces. The greyscale patterns are directly related to the intensity of fouling or the intensity of the residual deposits detected after the cleaning process. 


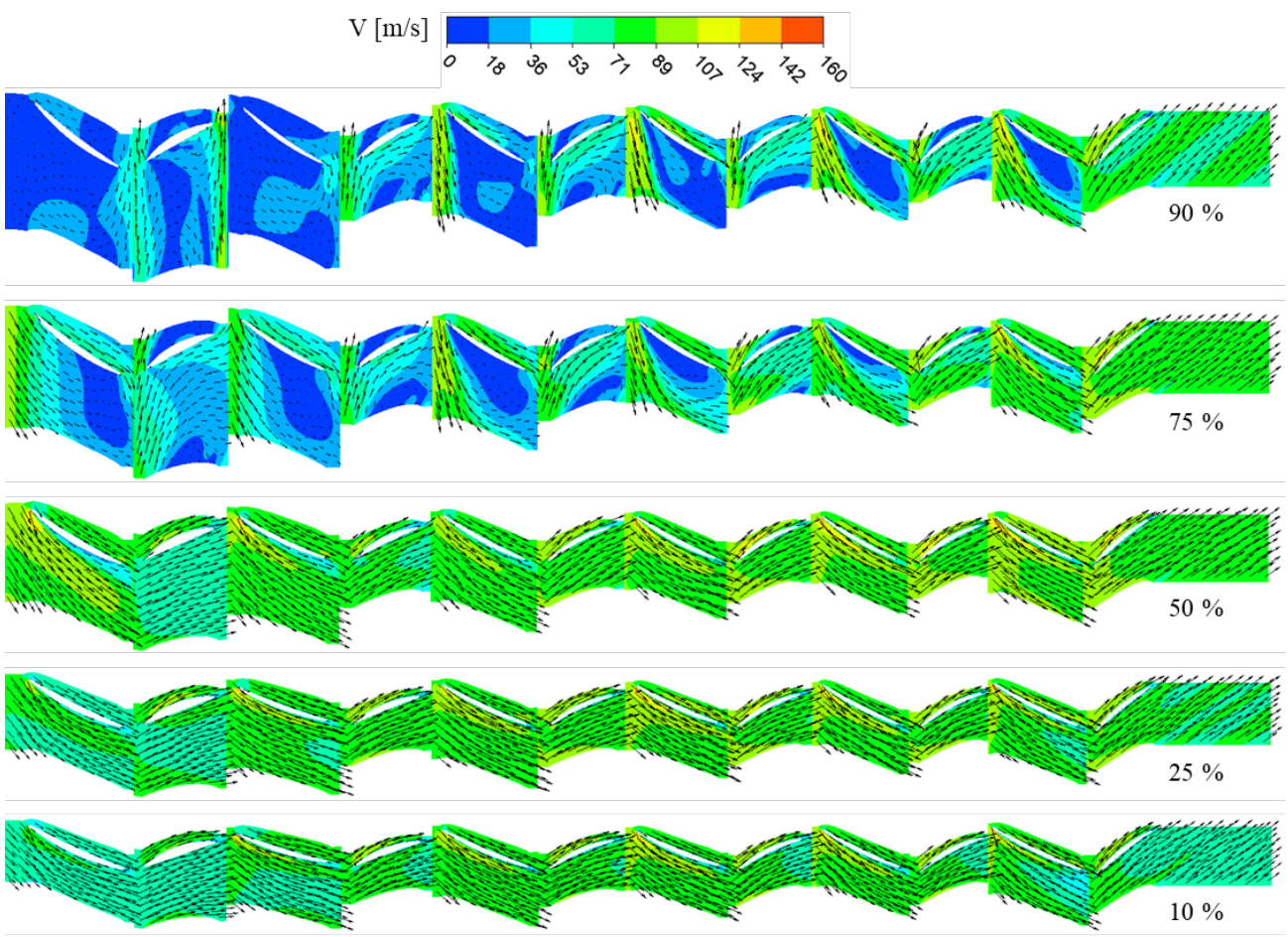

Fig. 4. Blade-to-blade velocity and vector plots for $10 \%, 25 \%, 50 \%, 75 \%$ and $90 \%$ of the blade span

The experimental results and the numerical data post-process have shown that smaller droplets were more effective at removing the soot deposits than coarser droplets. Due to the off-design conditions in which the compressor has been operated during the washing procedure, the formation of wider separation zones in correspondence with the airfoil surfaces may promote the diffusive motion of smaller droplets. The higher inertia, which characterizes coarser droplets, denies this mechanism of motion. This means that the washing equipment has to be designed to look at the contaminant type and the operating condition of the unit during the washing operation. Looking at the re-deposition process, bigger particles impact the first stages, collecting a higher amount of contaminants increases the re-deposition possibilities. By contrast, a great number of smaller particles can reach the rear stages without previous impacts, reducing the re-deposition process and increasing the overall washing effectiveness. Further development will be devoted to increasing the modeling of droplet impact on the compressor blade and vane surfaces.

\section{References}

1. M. Bohnet, Chem. Eng. Technol. 10, 113-125 (1987).

2. M. R. Maghami, H. Hizam, C. Gomes, M. A. Radzi, M. I. Rezadad, and S. Hajighorbani, Renew. Sust. Energ. Rev. 59, 1307-1316 (2016).

3. A. Suman, N. Casari, E. Fabbri, L. di Mare, F. Montomoli and M. Pinelli, Prog. Ener. Comb., 74, 103-151 (2019).

4. A. Suman, N. Casari, E. Fabbri, L. di Mare, F. Montomoli and M. Pinelli, J Eng. Gas Turb. Power, 141, 032601 (2019).

5. R. Kurz and K. Brun, J Eng. Gas Turb. Power, 134, 032401 (2012).

6. A. Suman, R. Kurz, N. Aldi, M. Morini, K. Brun, M. Pinelli and P.R. Spina, J Eng. Gas Turb. Power, 138, 012603 (2016). 
7. A. D. Mezheritsky and A. V. Sudarev, The mechanism of fouling and the cleaning technique in application to flow parts of the power generation plant compressors, 90-GT103, Proceedings of the ASME Turbo Expo, edit by ASME, NY (1990).

8. C. A: Perullo, T. Lieuwen, J. Barron, D. Grace and L. Angello, Evaluation of air filtration options for an industrial gas turbine, GT2015-43736, Proceedings of the ASME Turbo Expo, edit by ASME, NY (2015).

9. A. Suman, M. Morini, N. Aldi, N. Casari, M. Pinelli and P. R. Spina, J Turbomach. 139, 041005 (2017).

10. F.C. Mund and P. Pilidis, A review of gas turbine online washing systems, GT200453224, Proceedings of the ASME Turbo Expo, edit by ASME, NY (2004).

11. U. Igie, P. Pilidis, D. Fouflias, K. Ramsden, and P. Laskaridis, J. Turbomach., 136, 101001 (2014).

12. S76 - Performance and Operations Handbook. In The Australian Air Transport Pilot License (Helicopter); Civil Aviation Safety Authority, Australian Government: Woden, Australia (2013).

13. F. Costabile, W. Birmili, S. Klose, T. Tuch, B. Wehner, A. Wiedensohler, U. Franck, K. Konig and A. Sonntag, Atmos. Chem. Phys. 9, 3163-3195 (2009).

14. A. Vulpio, A. Suman, N. Casari, M. Pinelli, C. Appleby and S. Kyte, Washing effectiveness assessment of different cleaners on a small-scale multistage compressor, GT2021-59455, Proceedings of the ASME Turbo Expo edit by ASME, NY (2021). DOI: 10.1115/GT2021-59455

15. E. Syverud, and L.E. Bakken, J Turbomach., 129, pp. 136 - 142 (2007).

16. Allison Gas Turbine. Operation and Maintenance manual. Turboshaft models 250C18, A, B \& C. 15 September 1971, Rev. 16 June 1975. Detroit Diesel Allison, Division of General Motors Corporation, Indianapolis, IN 46206.

17. A. Suman, A. Vulpio, A. Fortini, E. Fabbri, N. Casari, M. Merlin and M. Pinelli, Int. J. Heat Mass Transf., 165, 120632 (2021).

18. C. A. Schneider, W. S. Rasband, and K. W: Eliceiri, Nat. Methods, 9(7), 671 (2012).

19. N. Casari, A. Fortini, M. Pinelli, A. Suman, A. Vulpio and N. Zanini, Measurement, Art. no. 110185. (2021) DOI: 10.1016/j.measurement.2021.110185

20. A. Vulpio, A. Suman, N. Casari, M. Pinelli, R. Kurz and K. Brun, K. J Eng. Gas Turb. Power, 143, 081005 (2021)

21. A. Suman, A. Vulpio, N. Casari, M. Pinelli, R. Kurz and K. Brun, K. J Eng. Gas Turb. Power, 143, 081006 (2021)

22. A. Suman, A. Vulpio, N. Casari, M. Pinelli, F. di Lillo and L. D'Amico, Powder Technol, 394, pp. 608-621 (2021)

23. A. Suman, A. Vulpio, N. Casari and M. Pinelli, Powder Technol, 394, pp. 597-607 (2021)

24. A. Suman, A. Vulpio, N. Casari and M. Pinelli, J Eng. Gas Turb. Power, GTP-21-1392 (2021). DOI: $10.1115 / 1.4051988$

25. N. Aldi, N. Casari, M. Pinelli, A. Suman, A. Vulpio and P. Saccenti. Performance modification of an erosion-damaged large-sized, GT2021-59832, Proceedings of the ASME Turbo Expo edit by ASME, NY (2021). DOI: 10.1115/GT2021-59832

26. A. Vulpio, A. Suman, N. Casari and M. Pinelli, A quantitative approach for the estimation of the fouling rate on the stationary parts of a multistage test compressor, GT2021-59449, Proceedings of the ASME Turbo Expo edit by ASME, Ny (2021). DOI: $10.1115 / \mathrm{GT} 2021-59449$

27. A. Vulpio, A. Suman, N. Casari and M. Pinelli, Aerospace, 81, art. no. 8030081 (2021).

28. N. Casari, M. Pinelli, P.R. Spina, A. Suman and A. Vulpio, J Eng. Gas Turb. Power, 143, $031020(2021)$ 